\title{
Supporting Information: Long-Range Supercoiling-Mediated RNA Polymerase Cooperation in Transcription
}

\author{
Alena Klindziuk and Anatoly Kolomeisky
}

March 1, 2021

\section{The Model}

There are six master equations that describe the dynamic transformations in the system,

$$
\begin{gathered}
\begin{array}{r}
\frac{d P_{\text {on }}(1 ; n, t)}{d t}=\alpha P_{\text {on }}(1 ; n-1, t)+(n+1) \beta P_{\text {on }}(1 ; n+1, t)+k_{\text {on }} \sum_{j=0}^{\infty} P_{j}(1 ; n, t) \\
+r_{\text {off }} P_{\text {on }}(2 ; n, t)-\left(\alpha+n \beta+k_{\text {off }}+r_{\text {on }}\right) P_{\text {on }}(1 ; n, t)
\end{array} \\
\begin{array}{r}
\frac{d P_{0}(1 ; n, t)}{d t}=(n+1) \beta P_{0}(1 ; n+1, t)+k_{\text {off }} P_{\text {on }}(1 ; n, t)+r_{\text {off }} P_{0}(2 ; n, t) \\
-\left(\alpha / y+n \beta+k_{\text {on }}+r_{\text {on }}\right) P_{0}(1 ; n, t)
\end{array} \\
\begin{array}{c}
\frac{d P_{j}(1 ; n, t)}{d t}=\alpha / y^{j} P_{j-1}(1 ; n-1, t)+(n+1) \beta P_{j}(1 ; n+1, t)+r_{\text {off }} P_{j}(2 ; n, t) \\
\frac{d P_{\text {on }}(2 ; n, t)}{d t}=2 \alpha P_{\text {on }}(2 ; n-1, t)+(n+1) \beta P_{\text {on }}(2 ; n+1, t)+k_{\text {on }} \sum_{j=0}^{\infty} P_{j}(2 ; n, t)
\end{array} \\
+r_{\text {on }} P_{\text {on }}(1 ; n, t)-\left(2 \alpha+n \beta+k_{\text {of }}\right) P_{j}(1 ; n, t)
\end{gathered}
$$




$$
\begin{gathered}
\frac{d P_{0}(2 ; n, t)}{d t}=(n+1) \beta P_{0}(2 ; n+1, t)+k_{\text {off }} P_{\text {on }}(2 ; n, t)+r_{\text {on }} P_{0}(1 ; n, t) \\
-\left((\alpha+\alpha / y)+n \beta+k_{\text {on }}+r_{\text {off }}\right) P_{0}(2 ; n, t)
\end{gathered}
$$

where $P_{\text {on }}(1 ; n, t)$ is defined as the probability density function of being in the ON state on the 1 RNAP plane with $n$ RNAs at time $t$, and $P_{j}(1 ; n, t)$ is the probability density function to find the system in the 1 RNAP plane and the state $j(j=0,1, \ldots)$ with $n$ RNA molecules at the time $t$. $P_{\text {on }}(2 ; n, t)$ and $P_{j}(2 ; n, t)$ have analogous definitions but for the 2 RNAP plane.

We define the stationary state (at $t \rightarrow 0$ ) probabilities as

$$
\begin{gathered}
P_{\text {on }}(1)=\sum_{n=0}^{\infty} P_{\text {on }}(1 ; n) \\
P_{\text {on }}(2)=\sum_{n=0}^{\infty} P_{\text {on }}(2 ; n) \\
P_{j}(1)=\sum_{n=0}^{\infty} P_{j}(1 ; n) \\
P_{j}(2)=\sum_{n=0}^{\infty} P_{j}(2 ; n) \\
P(1 ; n)=\sum_{j=0}^{\infty} P_{j}(1 ; n) \\
P(2 ; n)=\sum_{j=0}^{\infty} P_{j}(2 ; n) \\
P(1)=\sum_{n=0}^{\infty} \sum_{j=0}^{\infty} P_{j}(1 ; n) \\
P(2)=\sum_{n=0}^{\infty} \sum_{j=0}^{\infty} P_{j}(2 ; n) .
\end{gathered}
$$


The stationary state probability of being in the ON state with 1 RNAP is $P_{\text {on }}(1)$ and that of being in the $j$ state with 1 RNAP is $P_{j}(1)$. Likewise, the stationary state probabilities for the 2 RNAP plane are $P_{\text {on }}(2)$ in the ON state and $P_{j}(2)$ in the $j$ state. Note that once the system reaches the stationary state, there is going to be zero net flux between the corresponding macrostates.

In the stationary state limit, master equations can be rewritten as

$$
\begin{aligned}
& \alpha P_{o n}(1, n-1)+(n+1) \beta P_{o n}(1, n+1)+k_{o n} P(1, n)+r_{o f f} P_{o n}(2, n) \\
& -\left(\alpha+n \beta+k_{o f f}+r_{o n}\right) P_{o n}(1, n)=0 \\
& (n+1) \beta P_{0}(1, n+1)+k_{o f f} P_{o n}(1, n)+r_{o f f} P_{0}(2, n) \\
& -\left(n \beta+k_{o n}+r_{o n}+\alpha / y\right) P_{0}(1, n)=0 \\
& \alpha / y^{j} P_{j-1}(1, n-1)+(n+1) \beta P_{j}(1, n+1)+r_{o f f} P_{j}(2, n) \\
& -\left(n \beta+\alpha / y^{j+1}+k_{\text {on }}+r_{\text {on }}\right) P_{j}(1, n) \\
& 2 \alpha P_{\text {on }}(2, n-1)+(n+1) \beta P_{\text {on }}(2, n+1)+k_{\text {on }} P(2, n)+r_{\text {on }} P_{\text {on }}(1, n) \\
& -\left(2 \alpha+k_{o f f}+r_{o f f}+n \beta\right) P_{o n}(2, n)=0 \\
& (n+1) \beta P_{0}(2, n+1)+k_{\text {off }} P_{\text {on }}(2, n)+r_{\text {on }} P_{0}(1, n) \\
& -\left(\alpha+\alpha / y+k_{o n}+n \beta+r_{o f f}\right) P_{0}(2, n)=0 \\
& (n+1) \beta P_{j}(2, n+1)+\left(\alpha+\alpha / y^{j}\right) P_{j-1}(2, n-1)+r_{o n} P_{j}(1, n) \\
& -\left(\alpha+\alpha / y^{j+1}+n \beta+k_{o n}+r_{o f f}\right) P_{j}(2, n)=0 .
\end{aligned}
$$

Summing the stationary state equations over $n$, we obtain

$$
\begin{gathered}
k_{o n} P(1)+r_{o f f} P_{o n}(2)-\left(k_{o f f}+r_{o n}\right) P_{o n}(1)=0 \\
k_{o n} P(2)+r_{o n} P_{o n}(1)-\left(k_{o f f}+r_{o f f}\right) P_{o n}(2)=0 \\
k_{o f f} P_{o n}(1)+r_{o f f} P_{0}(2)-\left(\alpha / y+k_{o n}+r_{o n}\right) P_{0}(1)=0 \\
k_{o f f} P_{o n}(2)+r_{o n} P_{0}(1)-\left(\alpha+\alpha / y+k_{o n}+r_{o f f}\right) P_{0}(2)=0 \\
\frac{\alpha}{y^{j}} P_{j-1}(1)+r_{o f f} P_{j}(2)-\left(\frac{\alpha}{y^{j+1}}+k_{o n}+r_{o n}\right) P_{j}(1)=0 \\
\left(\alpha+\frac{\alpha}{y^{j}}\right) P_{j-1}(2)+r_{o n} P_{j}(1)-\left(\alpha+\frac{\alpha}{y^{j+1}}+k_{o n}+r_{o f f}\right) P_{j}(2)=0
\end{gathered}
$$




\section{Evaluating the Stationary Probabilities}

The sum of all stationary state probabilities is going to be equal to 1 due to normalization,

$$
P(1)+P(2)+P_{\text {on }}(1)+P_{\text {on }}(2)=1 .
$$

Using the zero-net-flux conditions

$$
k_{\text {on }}(P(1)+P(2))=k_{\text {off }}\left(P_{\text {on }}(1)+P_{\text {on }}(2)\right)
$$

and

$$
r_{\text {on }}\left(P_{\text {on }}(1)+P(1)\right)=r_{\text {off }}\left(P_{\text {on }}(2)+P(2)\right),
$$

as well as the normalization condition Eq. (27), the following relations can be derived:

$$
\begin{gathered}
P(1)+P(2)=\frac{\gamma}{1+\gamma} \\
P_{\text {on }}(1)+P_{\text {on }}(2)=\frac{1}{1+\gamma} \\
P_{\text {on }}(1)+P(1)=\frac{\sigma}{1+\sigma} \\
P_{\text {on }}(2)+P(2)=\frac{1}{1+\sigma}
\end{gathered}
$$

where $\gamma=k_{\text {off }} / k_{\text {on }}$ and $\sigma=r_{\text {off }} / r_{\text {on }}$.

We can now determine the probabilities $P_{\text {on }}(1), P_{\text {on }}(2), P(1)$, and $P(2)$ individually by solving a system of equations that includes the normalization condition (27), zero-net-flux conditions (28 - 29), and relevant steady state equations $(21-22)$, yielding

$$
\begin{gathered}
P_{\text {on }}(1)=\frac{\sigma}{(1+\gamma)(1+\sigma)} \\
P(1)=\frac{\gamma \sigma}{(1+\gamma)(1+\sigma)} \\
P_{\text {on }}(2)=\frac{1}{(1+\gamma)(1+\sigma)} \\
P(2)=\frac{\gamma}{(1+\gamma)(1+\sigma)} .
\end{gathered}
$$


The still unknown probabilities $P_{0}(1)$ and $P_{0}(2)$ can be found by solving the system of Eqs. $(23-24)$ :

$$
\begin{gathered}
k_{o f f} P_{o n}(1)+r_{o f f} P_{0}(2)-\left(\alpha / y+k_{o n}+r_{o n}\right) P_{0}(1)=0 \\
k_{o f f} P_{o n}(2)+r_{o n} P_{0}(1)-\left(\alpha+\alpha / y+k_{o n}+r_{o f f}\right) P_{0}(2)=0,
\end{gathered}
$$

and this results in

$$
\begin{gathered}
P_{0}(1)=\frac{k_{o f f}\left(k_{o n}+r_{o f f}+\alpha+\alpha / y\right) P_{o n}(1)}{\Gamma_{0}}+\frac{r_{o f f} k_{o f f} P_{o n}(2)}{\Gamma_{0}} \\
P_{0}(2)=\frac{k_{o f f}\left(k_{o n}+r_{o n}+\alpha / y\right) P_{o n}(2)}{\Gamma_{0}}+\frac{r_{o n} k_{o f f} P_{o n}(1)}{\Gamma_{0}}
\end{gathered}
$$

where

$$
\Gamma_{0}=\left(k_{o n}+\alpha / y\right)\left(k_{o n}+\alpha+\alpha / y\right)+r_{o n}\left(\alpha+\alpha / y+k_{o n}\right)+r_{o f f}\left(\alpha / y+k_{o n}\right) .
$$

Now for the case where $j>0$, to find the $P_{j}(1)$ and $P_{j}(2)$, we need to solve the system of equations $(25-26)$

$$
\begin{gathered}
\frac{\alpha}{y^{j}} P_{j-1}(1)+r_{o f f} P_{j}(2)-\left(\frac{\alpha}{y^{j+1}}+k_{o n}+r_{o n}\right) P_{j}(1)=0 \\
\left(\alpha+\frac{\alpha}{y^{j}}\right) P_{j-1}(2)+r_{o n} P_{j}(1)-\left(\alpha+\frac{\alpha}{y^{j+1}}+k_{o n}+r_{o f f}\right) P_{j}(2)=0,
\end{gathered}
$$

which leads to

$$
\begin{gathered}
P_{j}(1)=\frac{\alpha / y^{j}\left(\alpha+\alpha / y^{j+1}+k_{o n}+r_{o f f}\right) P_{j-1}(1)}{\Gamma_{j}}+\frac{r_{o f f}\left(\alpha+\alpha / y^{j}\right) P_{j-1}(2)}{\Gamma_{j}} \\
P_{j}(2)=\frac{\left(\alpha+\alpha / y^{j}\right)\left(\alpha / y^{j+1}+k_{o n}+r_{o n}\right) P_{j-1}(2)}{\Gamma_{j}}+\frac{r_{o n} \alpha / y^{j} P_{j-1}(1)}{\Gamma_{j}}
\end{gathered}
$$

where

$\Gamma_{j}=\left(k_{o n}+\alpha / y^{j+1}\right)\left(k_{o n}+\alpha+\alpha / y^{j+1}\right)+r_{o f f}\left(\alpha / y^{j+1}+k_{o n}\right)+r_{o n}\left(k_{o n}+\alpha+\alpha / y^{j+1}\right)$.

These equations allow us to explicitly evaluate all the stationary probabilities in the system. 


\section{Introducing the Generating Functions}

To proceed further, we introduce the generating functions that are defined as

$$
\begin{gathered}
g_{\text {on }}(1) \equiv \sum_{n=0}^{\infty} z^{n} P_{\text {on }}(1 ; n) ; \\
g_{\text {on }}(2) \equiv \sum_{n=0}^{\infty} z^{n} P_{\text {on }}(2 ; n) ; \\
g_{j}(1) \equiv \sum_{n=0}^{\infty} z^{n} P_{j}(1 ; n) ; \\
g_{j}(2) \equiv \sum_{n=0}^{\infty} z^{n} P_{j}(2 ; n) ; \\
g(1) \equiv \sum_{j=0}^{\infty} \sum_{n=0}^{\infty} z^{n} P_{j}(1 ; n) ; \\
g(2) \equiv \sum_{j=0}^{\infty} \sum_{n=0}^{\infty} z^{n} P_{j}(2 ; n) .
\end{gathered}
$$

Then, the original master equations $(1-6)$ can be rewritten in terms of the generating functions after multiplying each side by $z^{n}$ and summing over all $n$ :

$$
\begin{array}{r}
0=\left[\alpha(z-1)-k_{o f f}-r_{o n}\right] g_{o n}(1)+k_{o n} g(1)+r_{o f f} g_{o n}(2)-\beta(z-1) \frac{\partial g_{o n}(1)}{\partial z} \\
0=\left[2 \alpha(z-1)-k_{o f f}-r_{o f f}\right] g_{o n}(2)+k_{o n} g(2)+r_{o n} g_{o n}(1)-\beta(z-1) \frac{\partial g_{o n}(2)}{\partial z} \\
0=k_{o f f} g_{o n}(1)+r_{o f f} g_{0}(2)-\left(k_{o n}+r_{o n}+\alpha / y\right) g_{0}(1)-\beta(z-1) \frac{\partial g_{0}(1)}{\partial z} \\
0=k_{o f f} g_{o n}(2)+r_{o n} g_{0}(1)-\left(k_{o n}+r_{o f f}+\alpha+\alpha / y\right) g_{0}(2)-\beta(z-1) \frac{\partial g_{0}(2)}{\partial z} \\
0=\frac{\alpha(z-1)+\alpha}{y^{j}} g_{j-1}(2)-\left(\frac{\alpha}{y^{j+1}}+k_{o n}+r_{o n}\right) g_{j}(1)+r_{o f f} g_{j}(2) \\
-\beta(z-1) \frac{\partial g_{j}(1)}{\partial z}
\end{array}
$$




$$
\begin{array}{r}
0=\left[\left(\alpha+\frac{\alpha}{y^{j}}\right)(z-1)+\alpha+\frac{\alpha}{y^{j}}\right] g_{j-1}(2)+r_{\text {on }} g_{j}(1)-\left(\alpha+\frac{\alpha}{y^{j+1}}+k_{o n}+r_{o f f}\right) g_{j}(2) \\
-\beta(z-1) \frac{\partial g_{j}(2)}{\partial z} .
\end{array}
$$

\section{Calculating the First Moment}

Summing Eqs. (54 -59) together, we obtain

$$
\begin{aligned}
\frac{\partial}{\partial z}\left[g_{\text {on }}(1)+g_{\text {on }}(2)+g(1)+g(2)\right] & = \\
x\left(g_{\text {on }}(1)+2 g_{\text {on }}(2)\right. & \left.+\sum_{j=0}^{\infty}\left[\frac{g_{j}(1)}{y^{j+1}}+\left(1+\frac{1}{y^{j+1}}\right) g_{j}(2)\right]\right) .
\end{aligned}
$$

The first moment, which equal to the mean number of produced RNA, by definition is equal to

$$
\langle n\rangle=\sum_{n=0}^{\infty} n\left(P_{\text {on }}(1)+P_{\text {on }}(2)\right)+\sum_{j=0}^{\infty} \sum_{j=0}^{\infty} n\left(P_{j}(1, n)+P_{j}(2, n)\right) .
$$

Then, recognizing that definitions $(48$ - 53) imply that

$$
\begin{gathered}
z \frac{\partial g_{\text {on }}(1)}{\partial z} \equiv \sum_{n=0}^{\infty} n z^{n} P_{o n}(1, n) \\
z \frac{\partial g_{o n}(2)}{\partial z} \equiv \sum_{n=0}^{\infty} n z^{n} P_{o n}(2, n) \\
z \frac{\partial g_{j}(1)}{\partial z} \equiv \sum_{n=0}^{\infty} n z^{n} P_{j}(1, n) \\
z \frac{\partial g_{j}(2)}{\partial z} \equiv \sum_{n=0}^{\infty} n z^{n} P_{j}(2, n) \\
z \frac{\partial g(1)}{\partial z}=\sum_{j=0}^{\infty} \sum_{n=0}^{\infty} n z^{n} P_{j}(1, n) \\
z \frac{\partial g(2)}{\partial z} \equiv \sum_{j=0}^{\infty} \sum_{n=0}^{\infty} n z^{n} P_{j}(2, n)
\end{gathered}
$$


we can rewrite Eq. (61) as Eq. (60) for $z=1$, yielding

$$
\langle n\rangle=x\left[g_{\text {on }}(1)+2 g_{\text {on }}(2)+\sum_{j=0}^{\infty} \frac{1}{y^{j+1}} g_{j}(1)+\sum_{j=0}^{\infty}\left(1+\frac{1}{y^{j+1}}\right) g_{j}(2)\right] \text {. }
$$

And using the original generating function definitions (48-53), the first moment in terms of the known quantities is given by

$$
\langle n\rangle=x\left[P_{\text {on }}(1)+2 P_{\text {on }}(2)+\sum_{j=0}^{\infty} \frac{1}{y^{j+1}} P_{j}(1)+\sum_{j=0}^{\infty}\left(1+\frac{1}{y^{j+1}}\right) P_{j}(2)\right] \text {. }
$$

\section{Calculating the Second Moment}

Similar method is used to obtain the second moment. By definition, the second moment is:

$$
\left\langle n^{2}\right\rangle=\sum_{n=0}^{\infty} n^{2}\left(P_{\text {on }}(1)+P_{\text {on }}(2)\right)+\sum_{n=0}^{\infty} \sum_{j=0}^{\infty} n^{2}\left(P_{j}(1)+P_{j}(2)\right) .
$$

Since the definitions $(62-67)$ can be rewritten as

$$
\begin{gathered}
z^{2} \frac{\partial^{2} g_{o n}(1)}{\partial z^{2}}+z \frac{\partial g_{o n}(1)}{\partial z} \equiv \sum_{n=0}^{\infty} n^{2} z^{n} P_{o n}(1, n) \\
z^{2} \frac{\partial^{2} g_{o n}(2)}{\partial z^{2}}+z \frac{\partial g_{o n}(2)}{\partial z} \equiv \sum_{n=0}^{\infty} n^{2} z^{n} P_{o n}(2, n) \\
z^{2} \frac{\partial^{2} g_{j}(1)}{\partial z^{2}}+z \frac{\partial g_{j}(1)}{\partial z} \equiv \sum_{n=0}^{\infty} n^{2} z^{n} P_{j}(1, n) \\
z^{2} \frac{\partial^{2} g_{j}(2)}{\partial z^{2}}+z \frac{\partial g_{j}(2)}{\partial z} \equiv \sum_{n=0}^{\infty} n^{2} z^{n} P_{j}(2, n) \\
z^{2} \frac{\partial^{2} g(1)}{\partial z^{2}}+z \frac{\partial g(1)}{\partial z} \equiv \sum_{j=0}^{\infty} \sum_{n=0}^{\infty} n^{2} z^{n} P_{j}(1, n) \\
z^{2} \frac{\partial^{2} g(2)}{\partial z^{2}}+z \frac{\partial g(2)}{\partial z} \equiv \sum_{j=0}^{\infty} \sum_{n=0}^{\infty} n^{2} z^{n} P_{j}(2, n)
\end{gathered}
$$


the second moment is now

$$
\begin{aligned}
\left\langle n^{2}\right\rangle=\frac{\partial}{\partial z}\left[g_{\text {on }}(1)+g_{\text {on }}(2)+\right. & \left.\sum_{j=0}^{\infty}\left(g_{j}(1)+g_{j}(2)\right)\right]+ \\
& \frac{\partial^{2}}{\partial z^{2}}\left[g_{\text {on }}(1)+g_{\text {on }}(2)+\sum_{j=0}^{\infty}\left(g_{j}(1)+g_{j}(2)\right)\right] .
\end{aligned}
$$

Using equations (60) and (68), one can rewrite Eq. (77) as

$$
\left\langle n^{2}\right\rangle=\langle n\rangle+x \frac{\partial}{\partial z}\left(g_{\text {on }}(1)+2 g_{\text {on }}(2)+\sum_{j=0}^{\infty}\left[\frac{g_{j}(1)}{y^{j+1}}+\left(1+\frac{1}{y^{j+1}}\right) g_{j}(2)\right]\right) \text {. }
$$

To proceed further, we expand the generating functions

$$
\begin{aligned}
g_{\text {on }}(1) & \simeq P_{\text {on }}(1)+f_{\text {on }}(z-1)(1)+\ldots \\
g_{\text {on }}(2) & \simeq P_{\text {on }}(2)+f_{\text {on }}(z-1)(2)+\ldots \\
g_{j}(1) & \simeq P_{j}(1)+f_{j}(z-1)(1)+\ldots \\
g_{j}(2) & \simeq P_{j}(2)+f_{j}(z-1)(2)+\ldots \\
g(1) & \simeq P(1)+f(z-1)(1)+\ldots \\
g(2) & \simeq P(2)+f(z-1)(2)+\ldots
\end{aligned}
$$

where $f_{\text {on }}(1), f_{\text {on }}(2), f_{j}(1)$ and $f_{j}(2)$ are constant parameters. Differentiating the expansions $(79-84)$ with respect to $z$ cancels out the first term. Now using expansions $(79-84)$, Eq. (78) can be rewritten as:

$$
\left\langle n^{2}\right\rangle=\langle n\rangle+x\left[f_{\text {on }}(1)+2 f_{\text {on }}(2)+\sum_{j=0}^{\infty} \frac{f_{j}(1)}{y^{j+1}}+\sum_{j=0}^{\infty}\left(1+\frac{1}{y^{j+1}}\right) f_{j}(2)\right] .
$$

We can find parameters $f_{\text {on }}(1), f_{\text {on }}(2), f_{j}(1)$ and $f_{j}(2)$ using Eqs. (54 - 59). For that purpose, Eqs. (54 - 59) are rewritten in terms of the expansions (79 - 84) and similar powers of $z-1$ are collected. Thus, we obtain

$$
\begin{aligned}
& \left(\beta+k_{o f f}+r_{o n}\right) f_{\text {on }}(1)=k_{\text {on }} f(1)+\alpha P_{\text {on }}(1)+r_{\text {off } f} f_{\text {on }}(2) \\
& \left(\beta+k_{\text {off }}+r_{\text {off } f}\right) f_{\text {on }}(2)=k_{\text {on }} f(2)+2 \alpha P_{\text {on }}(2)+r_{\text {on }} f_{\text {on }}(1)
\end{aligned}
$$




$$
\begin{gathered}
\left(\beta+k_{\text {on }}+r_{\text {on }}+\alpha / y\right) f_{0}(1)=k_{\text {off } f} f_{\text {on }}(1)+r_{\text {off } f} f_{0}(2) \\
\left(b+k_{\text {on }}+r_{\text {off }}+\alpha+\alpha / y\right) f_{0}(2)=k_{\text {off }} f_{\text {on }}(2)+r_{\text {on }} f_{0}(1) \\
\left(\beta+k_{\text {on }}+r_{\text {on }}+\alpha / y^{j+1}\right) f_{j}(1)=\alpha / y^{j} P_{j-1}(1)+\alpha / y^{j} f_{j-1}(1)+r_{o f f} f_{j}(2) \\
\left(\beta+k_{\text {on }}+r_{\text {off }}+\alpha+\alpha / y^{j+1}\right) f_{j}(2)=\left(\alpha+\alpha / y^{j}\right) P_{j-1}(2)+\left(\alpha+\alpha / y^{j}\right) f_{j-1}(2)+r_{\text {on }} f_{j}(1)
\end{gathered}
$$

To find the expressions for the parameters $f_{\text {on }}(1), f_{\text {on }}(2), f_{j}(1)$ and $f_{j}(2)$ we need to solve a system of equations that includes (86), (87), the relevant condition on the flux, as well as the normalization condition. Note that the normalization condition is obtained here by rewriting the first moment definition (61) in terms of the generating functions (48-53), and then expanding the generating functions using the differentiated expansions (79-84). This system of equations is shown below:

$$
\begin{gathered}
\left(\beta+k_{o f f}+r_{o n}\right) f_{\text {on }}(1)=k_{\text {on }} f(1)+\alpha P_{\text {on }}(1)+r_{\text {off }} f_{\text {on }}(2) \\
\left(\beta+k_{\text {off }}+r_{\text {off } f}\right) f_{\text {on }}(1)=k_{\text {on }} f(2)+2 \alpha P_{\text {on }}(2)+r_{\text {on }} f_{\text {on }}(1) \\
r_{\text {on }}\left(f_{\text {on }}(1)+f(1)\right)=r_{\text {off }}\left(f_{\text {on }}(2)+f(2)\right) \\
f_{\text {on }}(1)+f_{\text {on }}(2)+f(1)+f(2)=\langle n\rangle
\end{gathered}
$$

The solutions for $f_{\text {on }}(1)$ and $f_{\text {on }}(2)$ are given by

$$
\begin{aligned}
& \begin{array}{l}
f_{\text {on }}(1)= \\
\frac{\alpha P_{o n}(1)\left(r_{o f f}+r_{o n}\right)\left(k_{o n}+k_{o f f}+\beta+r_{o f f}\right)+2 \alpha P_{o n}(2) r_{o f f}\left(r_{o f f}+r_{o n}\right)}{\left(r_{o f f}+r_{o n}\right)\left(\beta+k_{o f f}+k_{o n}+r_{o f f}+r_{o n}\right)\left(\beta+k_{o f f}+k_{o n}\right)}
\end{array} \\
& \frac{\langle n\rangle r_{o f f} k_{o n}\left(\beta+k_{o f f}+k_{o n}+r_{o f f}+r_{o n}\right)}{\left(r_{o f f}+r_{o n}\right)\left(\beta+k_{o f f}+k_{o n}+r_{o f f}+r_{o n}\right)\left(\beta+k_{o f f}+k_{o n}\right)} \\
& f_{\text {on }(2)=}= \\
& \frac{\alpha P_{o n}(1) r_{o n}\left(r_{o f f}+r_{o n}\right)+2 \alpha P_{o n}(2)\left(r_{o f f}+r_{o n}\right)\left(k_{o n}+k_{o f f}+\beta+r_{o n}\right)}{\left(r_{o f f}+r_{o n}\right)\left(\beta+k_{o f f}+k_{o n}+r_{o f f}+r_{o n}\right)\left(\beta+k_{o f f}+k_{o n}\right)} \\
& \frac{\langle n\rangle r_{o n} k_{o n}\left(k_{o n}+k_{o f f}+\beta+r_{o n}+r_{o f f}\right)}{\left(r_{o f f}+r_{o n}\right)\left(\beta+k_{o f f}+k_{o n}+r_{o f f}+r_{o n}\right)\left(\beta+k_{o f f}+k_{o n}\right)}
\end{aligned}
$$

Now, the parameters $f_{0}(1)$ and $f_{0}(2)$ for all $j$ can be found using equations derived from (88) and (89):

$$
f_{0}(1)=\frac{\left(\beta+k_{o n}+r_{o f f}+\alpha+\alpha / y\right) k_{o f f} f_{o n}(1)+r_{o f f} k_{o f f} f_{o n}(2)}{\left(\beta+k_{o n}+r_{o n}+\alpha / y\right)\left(\beta+k_{o n}+r_{o f f}+\alpha+\alpha / y\right)-r_{o f f} r_{o n}} ;
$$




$$
f_{0}(2)=\frac{\left(\beta+k_{o n}+r_{o n}+\alpha / y\right) k_{o f f} f_{o n}(2)+r_{o n} k_{o f f} f_{o n}(1)}{\left(\beta+k_{o n}+r_{o n}+\alpha / y\right)\left(\beta+k_{o n}+r_{o f f}+\alpha+\alpha / y\right)-r_{o f f} r_{o n}} .
$$

In the next step, the parameters $f_{j}(1)$ and $f_{j}(2)$ for all $j$ can be found using equations derived from (90) and (91):

$$
\begin{gathered}
f_{j}(1)=\frac{\left(\beta+k_{o n}+r_{o f f}+\alpha+\alpha / y^{j+1}\right)\left(\alpha / y^{j}\right) P_{j-1}(1)+r_{o f f}\left(\alpha+\alpha / y^{j}\right) P_{j-1}(2)}{\left(\beta+k_{o n}+r_{o n}+\alpha / y^{j+1}\right)\left(\beta+k_{o n}+r_{o f f}+\alpha+\alpha / y^{j+1}\right)-r_{o f f} r_{o n}} \\
+\frac{\left(\beta+k_{o n}+r_{o f f}+\alpha+\alpha / y^{j+1}\right)\left(\alpha / y^{j}\right) f_{j-1}(1)+r_{o f f}\left(\alpha+\alpha / y^{j}\right) f_{j-1}(2)}{\left(\beta+k_{o n}+r_{o n}+\alpha / y^{j+1}\right)\left(\beta+k_{o n}+r_{o f f}+\alpha+\alpha / y^{j+1}\right)-r_{o f f} r_{o n}},
\end{gathered}
$$

and

$$
\begin{aligned}
& f_{j}(2)=\frac{\left(\beta+k_{o n}+r_{o n}+\alpha / y^{j+1}\right)\left(\alpha+\alpha / y^{j}\right) P_{j-1}(2)+r_{o n}\left(\alpha / y^{j}\right) P_{j-1}(1)}{\left(\beta+k_{o n}+r_{o n}+\alpha / y^{j+1}\right)\left(\beta+k_{o n}+r_{o f f}+\alpha+\alpha / y^{j+1}\right)-r_{o f f} r_{o n}} \\
& +\frac{\left(\beta+k_{n}+r_{n}+\alpha / y^{j+1}\right)\left(\alpha+\alpha / y^{j}\right) f_{j-1}(2)+r_{o n}\left(\alpha / y^{j}\right) f_{j-1}(1)}{\left(\beta+k_{o n}+r_{o n}+\alpha / y^{j+1}\right)\left(\beta+k_{o n}+r_{o f f}+\alpha+\alpha / y^{j+1}\right)-r_{o f f} r_{o n}} .
\end{aligned}
$$

\section{One RNAP Case}

This limiting case can be viewed as the cooperative model with $r_{o n}=0$. Then the system will never go into the 2 RNAP plane, rendering $P_{o n}(2)$ and $P_{j}(2)$ all equal to zero, and all other calculations are significantly simplified. We have now

$$
\begin{gathered}
P_{o n}=\frac{1}{1+\gamma} \\
P_{0}=\frac{k_{f}}{\alpha / y+k_{n}} P_{o n} \\
P_{j}=\frac{\alpha / y^{j}}{\alpha / y^{j+1}+k_{n}} P_{j-1} \\
\langle n\rangle=x\left[P_{o n}+\sum_{j=0}^{\infty} \frac{1}{y^{j+1}} P_{j}\right]
\end{gathered}
$$

Also the second moment is :

$$
\left\langle n^{2}\right\rangle=\langle n\rangle+x\left[\frac{\partial g_{o n}}{\partial z}+\sum_{j=0}^{\infty} \frac{1}{y^{j+1}} \frac{\partial g_{j}}{\partial z}\right]
$$




$$
\left\langle n^{2}\right\rangle=\langle n\rangle+x\left[f_{\text {on }}+\sum_{j=0}^{\infty} \frac{1}{y^{j+1}} f_{j}\right]
$$

where

$$
f_{\text {on }}=\frac{\alpha P_{o n}+k_{n}\langle n\rangle}{\beta+k_{n}+k_{f}},
$$

and

$$
\begin{gathered}
f_{0}=\frac{k_{f} f_{\text {on }}}{\alpha / y+\beta+k_{n}} \\
f_{j}=\frac{\alpha / y^{j}}{\alpha / y^{j+1}+k_{n}+\beta} P_{j-1}+\frac{\alpha / y^{j}}{\alpha / y^{j+1}+k_{n}+\beta} f_{j-1}
\end{gathered}
$$

